\title{
Cerebrospinal Fluid Biomarkers for the Diagnosis of Prodromal Alzheimer's Disease in Amnestic Mild Cognitive Impairment
}

\author{
Jung Eun Park ${ }^{a, b}$ Kyu Yeong Choi ${ }^{c}$ Byeong C. Kim ${ }^{d}$ Seong-Min Choi ${ }^{d}$ \\ Min-Kyung Song ${ }^{d}$ Jang Jae Lee ${ }^{c}$ Jahae Kim $^{\mathrm{e}}$ Ho-Chun Song ${ }^{\mathrm{e}}$ \\ Hoo-Won Kim $^{f}$ Jung-Min Hag Eun Hyun Seo ${ }^{\text {h }}$ Woo Keun Song ${ }^{i}$ \\ Sung-Gyoo Park ${ }^{i}$ Jung Sup Lee ${ }^{a, b}$ Kun Ho Lee ${ }^{a, c}$ \\ aDepartment of Biomedical Science, Chosun University, Gwangju, Republic of Korea; \\ bBK21-PLUS Research Team for Bioactive Control Technology, Chosun University, Gwangju,

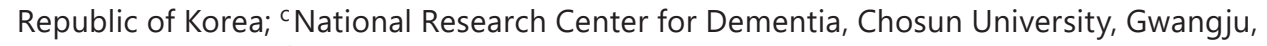 \\ Republic of Korea; ${ }^{d}$ Department of Neurology, Chonnam National University Hospital, \\ Gwangju, Republic of Korea; ' $D$ epartment of Nuclear Medicine, Chonnam National \\ University Hospital, Gwangju, Republic of Korea; ${ }^{f}$ Department of Neurology, School \\ of Medicine, Chosun University, Gwangju, Republic of Korea; ${ }^{9}$ Department of Nuclear \\ Medicine, School of Medicine, Chosun University, Gwangju, Republic of Korea; ${ }^{\text {hPremedical }}$ \\ Science, College of Medicine, Chosun University, Gwangju, Republic of Korea; 'School of Life \\ Science, Bioimaging, and Cell Dynamics Research Center, Gwangju Institute of Science and \\ Technology, Gwangju, Republic of Korea
}

\section{Keywords}

Alzheimer's disease - Prodromal Alzheimer's disease - Mild cognitive impairment .

Cerebrospinal fluid $\cdot$ Beta-amyloid $\cdot$ Biomarkers

\begin{abstract}
Background/Aims: Disease-modifying therapy for Alzheimer's disease (AD) has led to a need for biomarkers to identify prodromal $A D$ and very early stage of $A D$ dementia. We aimed to identify the cutoff values of cerebrospinal fluid (CSF) biomarkers for detecting prodromal AD. Methods: We assessed 56 patients with amnestic mild cognitive impairment (aMCl) who underwent lumbar puncture. Additionally, 87 healthy elderly individuals and 34 patients with AD dementia served as controls. Positron emission tomography was performed using florbetaben as a probe. We analyzed the concentration of $A \beta_{1-42}$, total tau protein (t-Tau), and tau protein phosphorylated at threonine 181 ( $p$-Tau $\left.{ }^{181}\right)$ in CSF with INNOTEST enzyme-linked immunosorbent assay. Results: For the detection of prodromal $A D$ in patients with $\mathrm{aMCl}$, the
\end{abstract}

J.E. Park and K.Y. Choi contributed equally to this work.

Byeong C. Kim

Department of Neurology

Chonnam National University Hospital, 42 Jebongro, Dongku

Gwangju 61469 (Republic of Korea)

E-Mail byeong.kim7@gmail.com
Kun Ho Lee

Department of Biomedical Science, Chosun University

309 Pilmundaero, Dongku

Gwangju 61452 (Republic of Korea)

E-Mail leekho@chosun.ac.kr 
cutoff values of CSF A $\beta_{1-42,}$ t-Tau, and $p$-Tau ${ }^{181}$ were $749.5 \mathrm{pg} / \mathrm{mL}, 225.6 \mathrm{pg} / \mathrm{mL}$, and $43.5 \mathrm{pg} /$ $\mathrm{mL}$, respectively. To discriminate prodromal $A D$ in patients with $\mathrm{aMCl}$, the t-Tau/A$\beta_{1-42}$ and $\mathrm{p}-\mathrm{Tau}^{181} / \mathrm{A} \beta_{1-42}$ ratios defined cutoff values at 0.298 and 0.059 , respectively. Conclusions: CSF biomarkers are very useful tools for the differential diagnosis of prodromal $A D$ in $\mathrm{AMCl}$ patients. The concentration of CSF biomarkers is well correlated with the stages of the AD spectrum.

(C) 2019 The Author(s)

Published by S. Karger AG, Basel

\section{Introduction}

Mild cognitive impairment (MCI) is defined as a transitional stage between normal aging and dementia and is based on stage 3 of the Global Deterioration Scale (GDS) [1]. In 2004, the Key Symposium in Sweden published consensus criteria for MCI to expand the concept of MCI beyond the memory domain [2,3]. MCI includes impairment in both memory and nonmemory cognitive domains $[2,4]$. MCI patients could be classified into two groups: amnestic MCI (aMCI) and nonamnestic MCI (naMCI) [2,3]. The classification by subtype could possibly predict the type of dementia that MCI patients may develop [2]. Because of the heterogeneity of the clinical presentation and underlying etiologies within the MCI group, there is no documented treatment for MCI. Furthermore, the clinical course of MCI is more heterogeneous than distinctive. In several previous longitudinal studies, aMCI was closely associated with a high risk of progression to Alzheimer's disease (AD) dementia [5-9]. MCI could provide significant information on the risk of becoming demented, and so it is a stage at which intervention could be effective in preventing conversion to dementia [3].

However, not all cases of aMCI progress to AD dementia, and some cases even return to normal [9]. It is conceivable that cognitive deficits can be attributable to a variety of medical and psychiatric illnesses instead of AD. Therefore, identifying MCI patients who will progress to AD dementia simply based on the clinical criteria for MCI may be inadequate. A new set of criteria has been proposed by the National Institute on Aging-Alzheimer's Association (NIA-AA) workgroup to further define MCI due to AD [10], or proposed by the International Working Group (IWG-2) to define prodromal AD [11], incorporating pathological biomarkers consistent with $\mathrm{AD}$. In addition, diagnostic research criteria for $\mathrm{AD}$ have recommended the use of cerebrospinal fluid (CSF) biomarkers to determine the etiology and prognosis in persons with MCI [10-13]. These new criteria would certainly improve the predictive value for the diagnosis of MCI. However, amyloid imaging, in particular, is expensive and not easily accessible to most primary care physicians, limiting its applicability.

Vandermeeren et al. [14] first demonstrated increased CSF tau concentration in AD. Since then, many reports have demonstrated that CSF biomarkers, in addition to neuropsychological tests or magnetic resonance imaging (MRI), increased the accuracy to predict progression to AD dementia in MCI patients [15-18]. CSF tau concentration especially may be considered as a potential biomarker for AD or a marker of neurodegeneration in MCI patients. However, the authors considered only conversion of AD dementia from MCI during the follow-up period, but not amyloidosis in MCI individuals at baseline. In addition, none of the previous reports have considered the IWG-2 guidelines in staging the $\mathrm{AD}$ spectrum.

The aims of this study were to determine the value of CSF biomarkers for differentiating prodromal AD from other MCI types in a group of patients with clinically diagnosed aMCI, and to assess whether they could serve as a gold standard as CSF biomarkers for prodromal AD. 


\section{Materials and Methods}

\section{Participants}

Subjects were prospectively recruited from two centers: Chonnam National University Hospital, and Chosun University Hospital including the National Research Center for Dementia in Gwangju, Korea. We prospectively collected data from 198 patients from September 2015 to August 2017. All subjects were examined by experienced neurologists and received a full dementia screening test, which included past history, neurological examination, laboratory and neuropsychological tests, and brain MRI. In addition, 177 of the 198 subjects underwent brain amyloid positron emission tomography (PET) and CSF analysis within 1 year from the screening test. The exclusion criterion was diagnosis of dementia due to causes not related to AD.

\section{Clinical and Neuropsychological Assessments}

Clinical assessments were undertaken according to the recommendations in the European Federation of Neurological Societies guideline [19]. All subjects were examined with a clinical interview including Clinical Dementia Rating [20,21] and GDS. Two activities of daily living (ADL) scales were included in the battery: the basic ADL and the instrumental ADL $[22,23]$. Demographic data included age, sex, and years of education. Global cognitive function was measured by the Korean version of the Mini-Mental State Examination (K-MMSE) [24, 25].

Neuropsychological assessment was conducted using the Seoul Neuropsychological Screening Battery, which measures attention, language and associated functions, visuospatial, memory, and frontal/executive functions [26]. Attention was evaluated using the digit span test (forward and backward). Language was evaluated using the Korean version of the Boston Naming Test. Visuospatial function was tested using the copy score of the Rey-Osterrieth Complex Figure Test. Memory was evaluated using the Seoul Verbal Learning Test (three learning-immediate recall trials of a 12-item list, a 20-min delayed recall trial, and recognition) and Rey-Osterrieth Complex Figure Test immediate recall and a 20-min delayed recall and recognition. Frontal/executive function was evaluated using the go-no-go test/contrasting program test, the Controlled Oral Word Association Test, and Stroop test. Cognitive impairment was defined as a $Z$-score $<-1.5$ standard deviation (normed for age and education) on at least one of the neuropsychological tests, which could be a memory, language, visuospatial function, attention, or frontal/executive test.

A routine blood test for dementia included complete blood count, biochemistry, thyroid function test, and syphilis serologic tests. For apolipoprotein E (APOE) genotyping, genomic DNA was extracted from 196 of the 198 participants using a commercially available kit (QuickGene DNA Whole Blood Kit, KURABO, catalog No.: DB-S). APOE genotyping was performed by SNaPshot-mediated SNP genotyping assays (Applied Biosystems) at DNALink (Seoul, South Korea).

Brain MRI was performed on all patients. The MRI protocol included a three-dimensional volumetric T1-weighted (T1W) sagittal scan, an axial T2W, an axial T2W fluid-attenuated inversion recovery, and a diffusion tensor image with slices angled parallel to the axis through anterior and posterior commissures and taken from the foramen magnum to the vertex. All patients who had structural brain lesions or severe subcortical white matter hyperintensities (WMH > D3P3; P3 = periventricular WMH's cap or band $\geq 10 \mathrm{~mm}$; D3 = maximum diameter of deep WMH $\geq 25 \mathrm{~mm}$ ) based on the Clinical Research Center for Dementia of South Korea WMH visual rating scale [27] were excluded. 


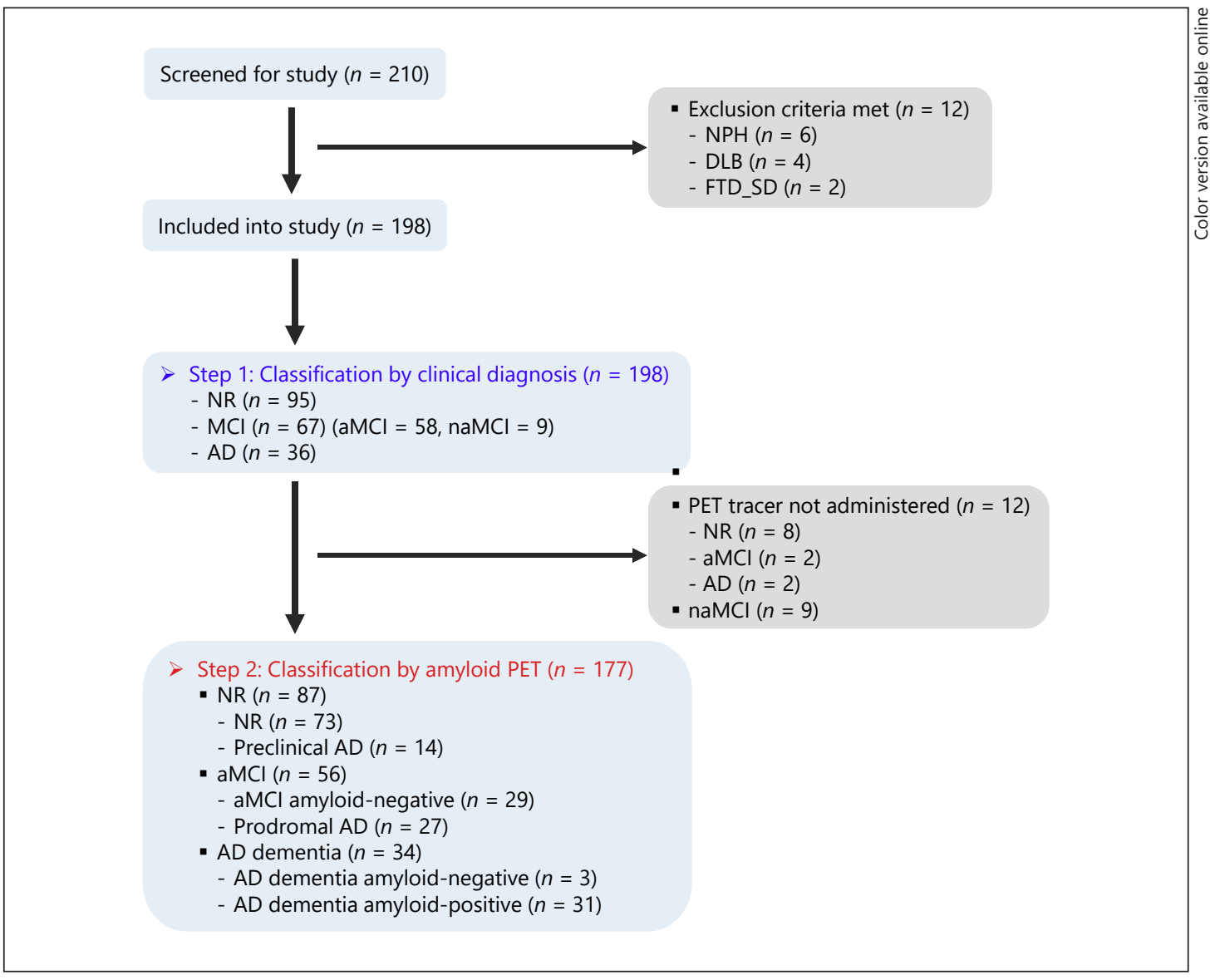

Fig. 1. Sample size flow chart of different stages. All subjects underwent CSF examination and amyloid PET scan on step 2, except for 12 subjects who withdrew from undergoing the amyloid PET scan. AD, Alzheimer's disease; aMCI, amnestic mild cognitive impairment; CSF, cerebrospinal fluid; DLB, dementia with Lewy bodies; FTD_SD, semantic variants of frontotemporal dementia; MCI, mild cognitive impairment; naMCI, nonamnestic mild cognitive impairment; NPH, normal-pressure hydrocephalus; NR, normal; PET, positron emission tomography.

\section{Classifications by Clinical Diagnosis}

We tried to screen 210 individuals for this study and excluded only 12 subjects: 6 who were diagnosed with normal-pressure hydrocephalus, 4 with dementia with Lewy bodies, and 2 with semantic variants of frontotemporal dementia.

The 198 subjects included in the study for the clinical diagnosis were classified into three groups: 95 normal elderly people, $67 \mathrm{MCI}$ subjects, and $36 \mathrm{AD}$ dementia patients (Fig. 1). Patients with AD dementia fulfilled the clinical criteria of probable AD dementia proposed by the NIA-AA or IWG-2 workgroups $[11,28]$. The diagnosis of MCI was made according to the MCI criteria proposed by the NIA-AA or IWG-2 on MCI $[2,3,10,11]$.

\section{Brain Florbetaben PET Imaging Acquisition and Analysis}

Brain PET images were acquired from participants 90-100 min after intravenous injection of a mean dose of $303 \mathrm{MBq} 20 \%$ florbetaben, a fluorine-18-labeled stilbene derivative with the trade name of NeuraCeq [29], according to a standardized acquisition and imaging protocol in two hospitals. The florbetaben radiosynthesis, PET imaging scan, and 
Park et al.: CSF Biomarkers for the Diagnosis of Prodromal AD

Fig. 2. CSF biomarker change during the progression of $\mathrm{AD}$ spectrum from amyloid-negative normal to amyloid-positive AD dementia. CSF $A \beta_{1-42}$ levels are decreasing, and t-Tau and p-Tau levels are increasing during $\mathrm{AD}$ progression. Number of subjects: amyloid-negative normal $n=73$, preclinical $\mathrm{AD} n=14$, prodromal AD $n=27$, and amyloid-positive AD dementia $n=31$. $*$ Statistically significant difference between the indicated group and the normal amyloid-negative group. AD, Alzheimer's disease; CSF, cerebrospinal fluid; $p$-Tau, phosphorylated tau protein; t-Tau, total tau protein.

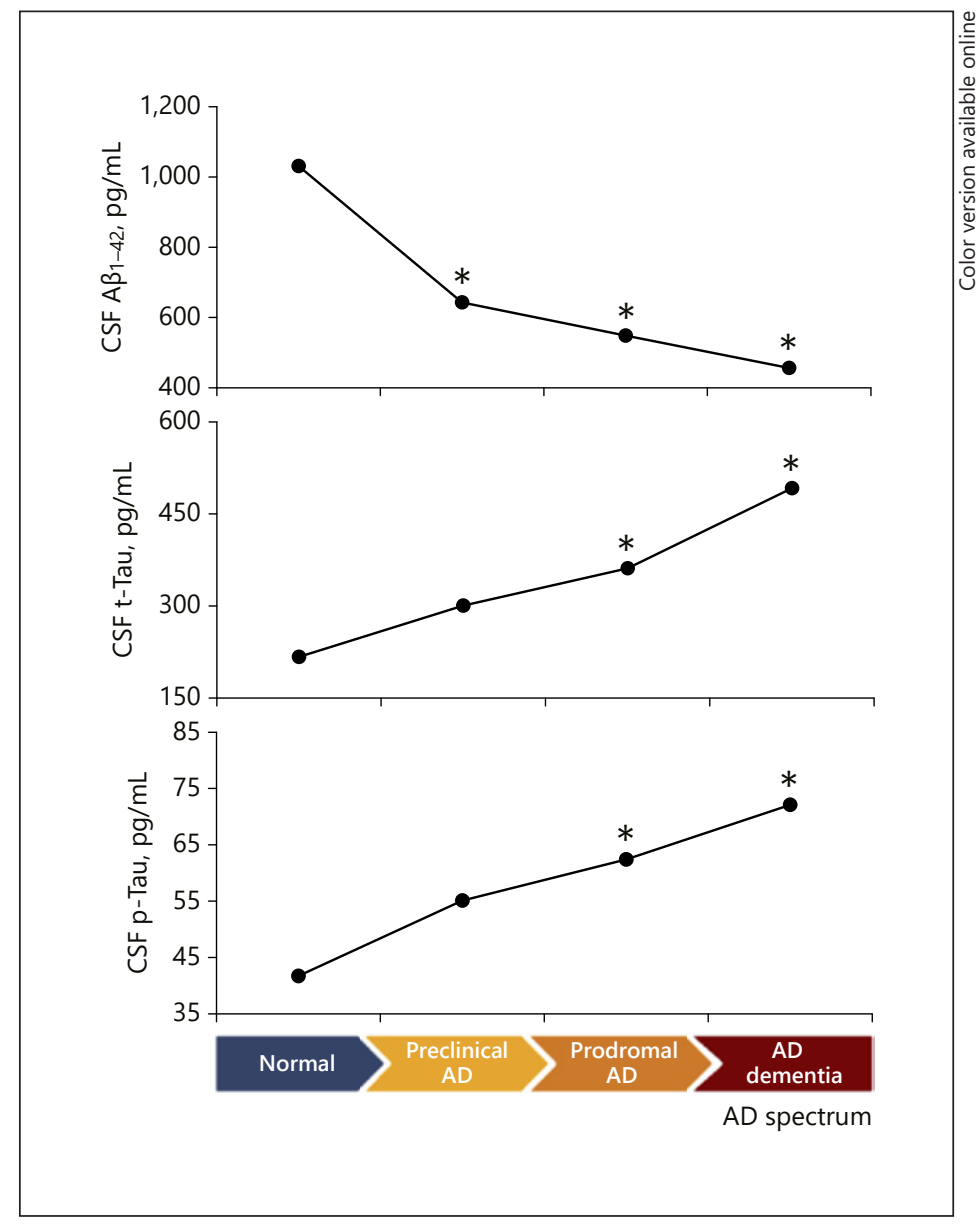

data processing were performed as previously described in detail [30, 31]. PET data were acquired with a Discovery STE PET-CT scanner (GE Medical Systems, Milwaukee, WI, USA).

The visual assessment of florbetaben PET images was performed in transaxial images using a gray scale by trained readers (J. Kim and H.-C. Song). Each brain region (frontal cortex, lateral temporal cortex, parietal cortex, and posterior cingulate cortex/precuneus) was visually assessed and scored according to the brain beta-amyloid plaque load (BAPL) scoring system for each PET scan. BAPL score: 1 = no beta-amyloid load, 2 = minor beta-amyloid load, 3 = significant beta-amyloid load. BAPL scores of 1 are classified as beta-amyloid-negative PET scan, and BAPL scores of 2 and 3 as beta-amyloid-positive PET scan [32].

\section{Classifications by Amyloid PET}

Twenty-one of the 198 subjects who were classified by clinical diagnosis were excluded. Of these, 9 subjects had naMCI. The other 12 subjects ( 8 normal, 2 aMCI, and 2 AD dementia) were not administered amyloid PET tracer. Finally, 177 subjects were selected ( 87 normal subjects, 56 aMCI patients, and $34 \mathrm{AD}$ dementia patients) (Fig. 1; Table 1).

Patients who were diagnosed with prodromal AD in this classification met the IWG-2 guidelines based on amyloid PET results [11]. In this classification, 14 of 87 normal elderly people were amyloid-positive, named "preclinical AD," 27 of 56 aMCI patients, named "prodromal AD," and 31 of $34 \mathrm{AD}$ dementia patients were amyloid-positive (Fig. 1; Table 2). 
Park et al.: CSF Biomarkers for the Diagnosis of Prodromal AD

Table 1. Clinical characteristics and CSF data of subjects with a clinical diagnosis ( $n=177)$

\begin{tabular}{|c|c|c|c|c|c|}
\hline Characteristics & Total & Normal & aMCI & AD dementia & $p$ value \\
\hline Subjects, $n$ & 177 & 87 & 56 & 34 & \\
\hline Age, years & 177 & $71.7(5.8)$ & $71.5(8.0)$ & $69.4(6.0)$ & 0.215 \\
\hline Education, years & 177 & $9.6(5.0)$ & $9.0(4.7)$ & $7.0(3.9)^{\mathrm{a}}$ & 0.026 \\
\hline Female sex & 177 & $46(52.8 \%)$ & $26(46.4 \%)$ & $19(55.9 \%)$ & 0.637 \\
\hline APOE $\varepsilon 4$ carrier status & 175 & $22(25.3 \%)$ & $24(42.9 \%)$ & $20(58.8 \%)^{a, b}$ & 0.002 \\
\hline K-MMSE score, points & 177 & $26.7(2.6)$ & $25.5(3.6)$ & $18.5(5.4)^{\mathrm{a}, \mathrm{b}}$ & $<0.001$ \\
\hline CDR & 177 & $0.3(0.2)$ & $0.5(0.0)^{\mathrm{a}}$ & $0.8(0.4)^{\mathrm{a}, \mathrm{b}}$ & $<0.001$ \\
\hline CDR sum of boxes & 177 & $0.5(0.6)$ & $1.3(0.7)^{\mathrm{a}}$ & $4.6(2.6)^{a, b}$ & $<0.001$ \\
\hline GDS & 177 & $1.6(0.5)$ & $2.9(0.3)^{\mathrm{a}}$ & $3.9(0.9)^{\mathrm{a}, \mathrm{b}}$ & $<0.001$ \\
\hline B-ADL & 177 & $20.0(0.0)$ & $20.0(0.2)$ & $19.0(1.7)^{\mathrm{a}, \mathrm{b}}$ & $<0.001$ \\
\hline I-ADL & 177 & $0.04(0.09)$ & $0.18(0.16)^{\mathrm{a}}$ & $0.60(0.19)^{\mathrm{a}, \mathrm{b}}$ & $<0.001$ \\
\hline $\mathrm{CSF} A \beta_{1-42}, \mathrm{pg} / \mathrm{mL}$ & 177 & $982.4(236.5)$ & $771.0(317.0)^{\mathrm{a}}$ & $559.0(311.0)^{\mathrm{a}, \mathrm{b}}$ & $<0.001$ \\
\hline $\mathrm{CSF} A \beta_{1-40}, \mathrm{pg} / \mathrm{mL}$ & 177 & $10,120.1(4,709.0)$ & $8,120.6(3,532.1)^{\mathrm{a}}$ & $7,311.6(3,795.3)^{\mathrm{a}}$ & 0.001 \\
\hline CSF t-Tau, pg/mL & 177 & $247.9(105.2)$ & 303.8 (229.7) & $487.5(218.6)^{a, b}$ & $<0.001$ \\
\hline CSF p-Tau ${ }^{181}, \mathrm{pg} / \mathrm{mL}$ & 177 & $47.6(17.9)$ & $53.1(29.9)$ & $72.6(28.1)^{a, b}$ & $<0.001$ \\
\hline
\end{tabular}

Data are presented as mean (standard deviation) or $n(\%)$ unless indicated otherwise. A $\beta$, beta-amyloid protein; AD, Alzheimer's disease; aMCI, amnestic mild cognitive impairment; APOE, apolipoprotein E; B-ADL, basic activities of daily living; CDR, Clinical Dementia Rating; CSF, cerebrospinal fluid; GDS, Global Deterioration Scale; I-ADL, instrumental activities of daily living; K-MMSE, Korean version of the Mini-Mental State Examination; p-Tau ${ }^{181}$, tau protein phosphorylated at threonine 181 ;

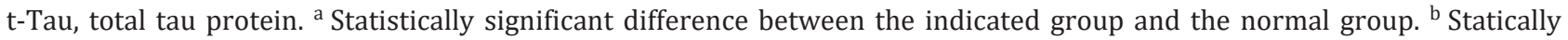
significant difference between the aMCI and the AD dementia group.

\section{CSF Analysis}

CSF samples were obtained by lumbar puncture with aseptic technique at the L3-L4 or L4-L5 intervertebral spinous process space, using a 22- or 21-gauge needle, between 8 a.m. and 10 a.m. CSF was collected in Falcon polypropylene tubes (BD Biosciences, Franklin Lakes, NJ, USA), and the first 2-3 mL of CSF were analyzed for routine chemical parameters, including cell count as well as glucose and total protein concentrations. Within $15 \mathrm{~min}$, the remaining CSF samples were centrifuged for $10 \mathrm{~min}$ at $2,000 \mathrm{rpm}$ at $4{ }^{\circ} \mathrm{C}$ to remove cells, aliquoted to 0.45 - mL-samples in polypropylene tubes, and then stored at $-80{ }^{\circ} \mathrm{C}$ until analysis. CSF biomarker concentrations of $A \beta_{1-42}, A \beta_{1-40}$, total tau protein (t-Tau), and tau protein phosphorylated at threonine $181\left(\mathrm{p}-\mathrm{Tau}^{181}\right)$ were measured in duplicate using a commercially available double antibody sandwich enzyme-linked immunosorbent assay kit (INNOTEST; Fujirebio, Gent, Belgium) according to the manufacturer's protocol. Absorbance was measured at $450 \mathrm{~nm}$ using a SpectraMAX M3 microplate reader (Molecular Devices, San Jose, CA, USA). We calculated derived ratios from single biomarkers including $A \beta_{1-42} / A \beta_{1-40}$, t-Tau/A $\beta_{1-42}$, and $\mathrm{p}-\mathrm{Tau}^{181} / \mathrm{A} \beta_{1-42}$ ratios. The team involved in CSF analyses was blinded to the clinical diagnosis.

\section{Statistical Analyses}

For the statistical analyses, the IBM SPSS software version 24.0 for Windows (IBM, Armonk, NY, USA) was used. The participants were grouped by the states of the clinical diagnosis, the amyloidosis on amyloid PET, and the combination of them for the statistical analysis. The normality of the continuous variables was tested using the Shapiro-Wilk test. The clinical and demographic characteristics of the subgroups were compared among the clinically defined three groups and between the defined two subgroups using one-way analysis of variance and $\chi^{2}$ tests for continuous and categorical variables, respectively. The levels of CSF 


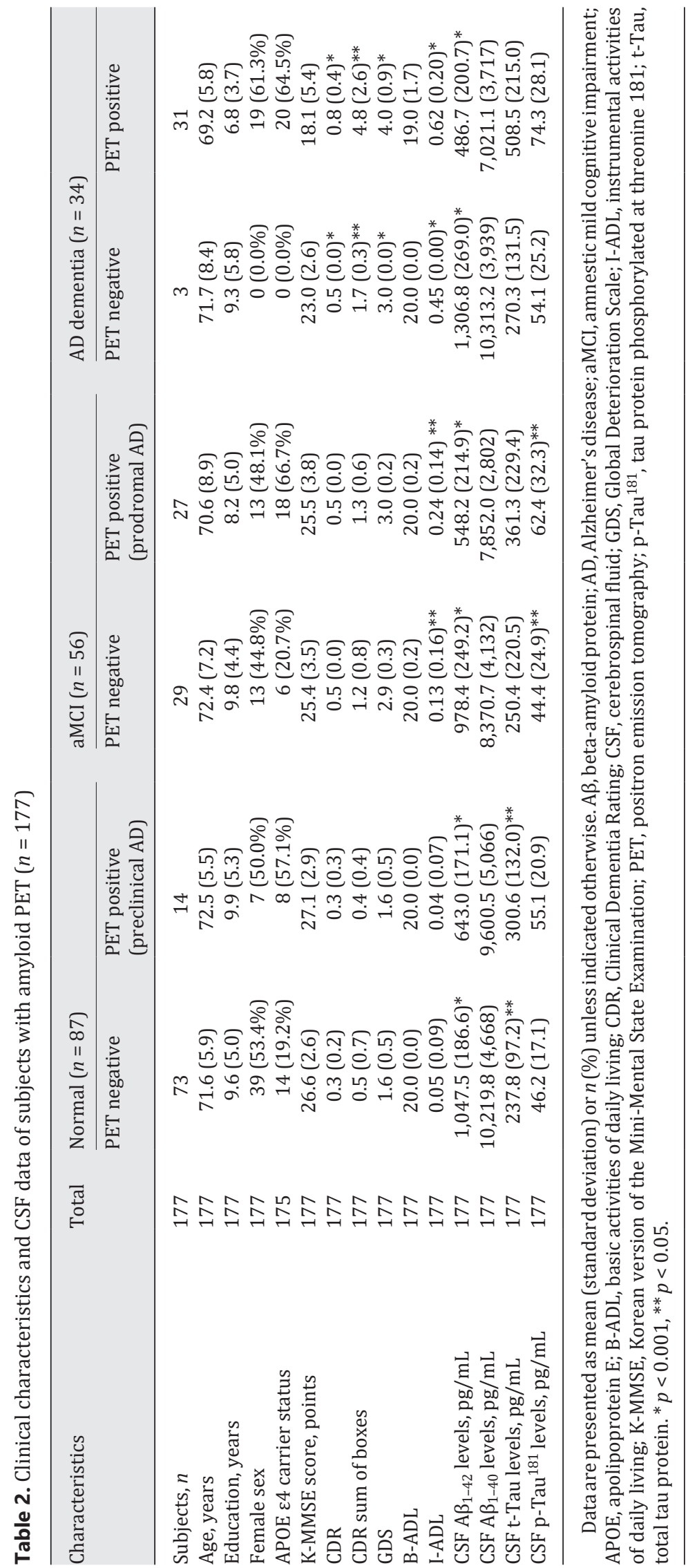


biomarkers including $A \beta_{1-42}, A \beta_{1-40}$, t-Tau, and p-Tau ${ }^{181}$ as well as scores on neuropsychological, clinical, and functional measures were compared between groups using analysis of covariance with post hoc analyses (LSD test).

Receiver operating characteristic (ROC) curves were depicted with the true-positive fraction (sensitivity) and the false-positive fraction (1 - specificity) by SPSS software 24.0. The area under the curve (AUC) was estimated from each ROC curve using an empirical method (confidence interval at 95\%) [33], because it does not make the strong normality assumptions and was used as an index of test performance. The specificities and sensitivities of each CSF biomarker level, the ratios of CSF biomarkers, or the combinations of CSF biomarkers for the indicated pairs were optimized using the ROC curve. In addition, the cutoffs for each biomarker were the scores that yielded the highest sum of sensitivity and specificity. The sensitivity and specificity were calculated for each cutoff value. A $p$ value $<0.05$ was considered statistically significant in the analysis.

\section{Results}

\section{Characteristics and CSF Data of Study Participants with Clinical Diagnosis}

The clinical characteristics and CSF data of 177 subjects are presented in Table 1. There were 87 normal elderly people, $56 \mathrm{aMCI}$ patients, and $34 \mathrm{AD}$ dementia patients. The mean age of the normal, aMCI, and AD dementia subjects was $71.7 \pm 5.8,71.5 \pm 8.0$, and $69.4 \pm 6.0$ years, respectively. Age and sex were not statistically different in these three groups (Table 1). Education level was statistically lowest in AD dementia patients. APOE $\varepsilon 4$ allele carrier status was observed in 22 normal subjects (25.3\%), in 24 patients with aMCI (42.9\%), and in 20 patients with AD dementia (58.8\%). The frequency of APOE $\varepsilon 4$ allele was significantly higher in $\mathrm{AD}$ dementia patients than in normal subjects and in aMCI patients $(p<0.001)$. The K-MMSE scores were significantly lower in AD dementia patients $(18.5 \pm 5.4)$ than in aMCI patients $(25.5 \pm 3.6)$ and in normal subjects $(26.7 \pm 2.6)(p<0.001)$. The Clinical Dementia Rating and sum of box scores, GDS, and instrumental ADL were statistically lower in the normal group compared to the $\mathrm{aMCI}$ and $\mathrm{AD}$ dementia groups $(p<0.001)$ (Table 1$)$.

The CSF concentrations of $A \beta_{1-42}$ were highest in the normal group (982.4 $\pm 236.5 \mathrm{pg}$ / $\mathrm{mL})$ compared to the aMCI $(771.0 \pm 317.0 \mathrm{pg} / \mathrm{mL})$ and AD dementia $(559.0 \pm 311.0 \mathrm{pg} / \mathrm{mL})$ groups $(p<0.001)$. CSF A $\beta_{1-42}$ levels can be different in aMCI, AD dementia, or normal subjects. The CSF concentrations of t-Tau were highest in the AD dementia group (487.5 $\pm 218.6 \mathrm{pg}$ / $\mathrm{mL})$ compared to the normal $(247.9 \pm 105.2 \mathrm{pg} / \mathrm{mL})$ and $\mathrm{aMCI}(303.8 \pm 229.7 \mathrm{pg} / \mathrm{mL})$ groups $(p<0.001)$. CSF t-Tau levels can also be different in AD dementia, aMCI, or normal subjects. The CSF concentration of $\mathrm{p}$-Tau ${ }^{181}$ in the AD dementia group was $72.6 \pm 28.0 \mathrm{pg} / \mathrm{mL}$, which was statistically higher than in the normal $(47.6 \pm 17.9 \mathrm{pg} / \mathrm{mL})$ or aMCI $(53.1 \pm 29.9 \mathrm{pg} / \mathrm{mL})$ groups. CSF p-Tau ${ }^{181}$ levels can also be different in AD dementia compared to normal or aMCI subjects (Table 1 ).

\section{Characteristics and CSF Data of Study Participants with Amyloid PET}

On the basis of amyloidosis, 177 subjects were classified into 87 normal elderly people (73 amyloid-negative and 14 amyloid-positive), 56 of the aMCI group (29 amyloid-negative and 27 amyloid-positive), and 34 of the AD dementia group ( 3 amyloid-negative and 31 amyloid-positive) (Table 2). APOE $\varepsilon 4$ allele carrier status was observed in 8 normal amyloidpositive subjects (preclinical AD, 57.1\%), in 18 amyloid-positive aMCI patients (prodromal $\mathrm{AD}, 66.7 \%)$, and in 20 amyloid-positive $\mathrm{AD}$ dementia patients (64.5\%). Statistically, CSF $\mathrm{A} \beta_{1-42}$ protein levels were lower in prodromal $\mathrm{AD}(548.2 \pm 214.9 \mathrm{pg} / \mathrm{mL})$ compared with 
Park et al.: CSF Biomarkers for the Diagnosis of Prodromal AD

Table 3. Sensitivity and specificity of CSF biomarkers in prodromal AD of the aMCI group

\begin{tabular}{|c|c|c|c|c|c|c|c|}
\hline \multirow[t]{2}{*}{ Characteristics } & \multicolumn{7}{|c|}{ Prodromal AD $(n=27)$ versus amyloid-negative aMCI $(n=29)$} \\
\hline & cutoff & $\mathrm{SE}, \%$ & $\mathrm{SP}, \%$ & AUC & PPV, \% & NPV, \% & $p$ value \\
\hline $\mathrm{CSF} A \beta_{1-42}, \mathrm{pg} / \mathrm{mL}$ & $<749.5$ & 85.2 & 86.2 & 0.908 & 85.2 & 86.2 & $<0.001$ \\
\hline CSF t-Tau, pg/mL & $>225.6$ & 63.0 & 65.5 & 0.692 & 63.0 & 65.5 & 0.087 \\
\hline CSF p-Tau ${ }^{181}, \mathrm{pg} / \mathrm{mL}$ & $>43.5$ & 63.0 & 69.0 & 0.678 & 65.4 & 66.7 & 0.036 \\
\hline $\mathrm{A} \beta_{1-42} / \mathrm{A} \beta_{1-40}$ & $<0.095$ & 85.2 & 79.3 & 0.857 & 79.3 & 85.2 & 0.001 \\
\hline $\mathrm{t}-\mathrm{Tau} / \mathrm{A} \beta_{1-42}$ & $>0.298$ & 77.8 & 82.8 & 0.853 & 80.8 & 80.0 & 0.004 \\
\hline $\mathrm{p}-\mathrm{Tau}{ }^{181} / \mathrm{A} \beta_{1-42}$ & $>0.059$ & 81.5 & 86.2 & 0.891 & 84.6 & 83.3 & 0.001 \\
\hline \multicolumn{8}{|l|}{ Combination } \\
\hline $\mathrm{A} \beta_{1-42}+\mathrm{p}-\mathrm{Tau}^{181}$ & & 92.6 & 89.7 & 0.935 & 89.3 & 92.9 & \\
\hline $\mathrm{p}-\mathrm{Tau}^{181} / \mathrm{A} \beta_{1-42}+\mathrm{t}-\mathrm{Tau}$ & & 92.6 & 86.2 & 0.930 & 81.5 & 93.1 & \\
\hline
\end{tabular}

$\mathrm{A} \beta$, beta-amyloid protein; AD, Alzheimer's disease; aMCI, amnestic mild cognitive impairment; AUC, area under the curve; CSF, cerebrospinal fluid; NPV, negative predictive value; PPV, positive predictive value; p-Tau $^{181}$, tau protein phosphorylated at threonine 181; SE, sensitivity; SP, specificity; t-Tau, total tau protein.

aMCI amyloid-negative patients $(978.4 \pm 249.2 \mathrm{pg} / \mathrm{mL})$, whereas CSF p-Tau ${ }^{181}$ levels were higher in prodromal $\mathrm{AD}(62.4 \pm 32.3 \mathrm{pg} / \mathrm{mL})$ compared with amyloid-negative aMCI patients $(44.4 \pm 24.9 \mathrm{pg} / \mathrm{mL})$. However, CSF A $\beta_{1-40}$ protein and CSF t-Tau levels showed no statistical difference between prodromal $\mathrm{AD}$ and amyloid-negative aMCI patients. Preclinical AD patients had statistically lower CSF $A \beta_{1-42}(643.0 \pm 171.1 \mathrm{pg} / \mathrm{mL})$ and higher t-Tau $(300.6 \pm$ $132.0 \mathrm{pg} / \mathrm{mL}$ ) compared with normal amyloid-negative subjects $(1,047.5 \pm 186.6$ and 237.8 $\pm 97.2 \mathrm{pg} / \mathrm{mL}$, respectively); however, CSF p-Tau ${ }^{181}$ showed no statistical difference between preclinical AD patients and amyloid-negative normal subjects (Table 2). All three CSF biomarkers defined and were related to the status of the AD spectrum (from normal to AD dementia) according to the IWG-2, with CSF $A \beta_{1-42}$ protein levels decreasing, and others increasing during $\mathrm{AD}$ progression (Fig. 2). The analysis revealed that the neuropsychological tests were similar between the amyloid-positive and -negative subjects in each of the three groups (normal, aMCI, AD dementia) (online suppl. Table 1; for all online suppl. material, see www.karger.com/doi/10.1159/000496920).

CSF Biomarkers between Prodromal AD and Amyloid-Negative aMCI Patients in the aMCI Group, and between Amyloid-Positive AD Dementia Patients and Amyloid-Negative Normal Subjects

The analysis of CSF biomarkers revealed that they differed significantly between prodromal $\mathrm{AD}$ and amyloid-negative aMCI. We generated ROC curves to identify CSF biomarkers that differentiated the two groups. The cutoff values that yielded the best index for prodromal AD were $749.5 \mathrm{pg} / \mathrm{mL}$ for $\mathrm{A} \beta_{1-42}, 225.6 \mathrm{pg} / \mathrm{mL}$ for t-Tau, $43.5 \mathrm{pg} / \mathrm{mL}$ for $\mathrm{p}$-Tau ${ }^{181}$, 0.095 for $A \beta_{1-42} / A \beta_{1-40}, 0.298$ for $t-T a u / A \beta_{1-42}$, and 0.059 for $p-T a u^{181} / A \beta_{1-42}$. The AUCs, the sensitivity, and the specificity of each CSF biomarker are shown in Table 3. The ROC curve and AUC for prodromal $\mathrm{AD}$ and amyloid-negative aMCI are illustrated in online supplementary Figure 1 . The cutoff ratio indicative of prodromal $\mathrm{AD}$ from amyloid-negative aMCI was set at $A \beta_{1-42} / A \beta_{1-40}<0.095$, t-tau $/ A \beta_{1-42}>0.298$, and $p-T a{ }^{181} / A \beta_{1-42}>0.059$ based on the amyloidosis with florbetaben PET (Table 3).

We applied the cutoff values to differentiate preclinical AD patients $(n=14)$, prodromal AD patients $(n=27)$, and amyloid-positive AD dementia patients $(n=31)$ from normal amyloid-negative subjects $(n=73)$. The cutoff values for amyloid-positive AD dementia 
Table 4. Diagnostic cutoff values in differentiating patients with preclinical AD $(n=14)$, prodromal AD ( $n=27)$, and amyloidpositive AD dementia ( $n=31$ ) from normal amyloid-negative subjects $(n=73)$

\begin{tabular}{|c|c|c|c|c|c|c|c|c|c|c|c|c|}
\hline & \multicolumn{4}{|c|}{ Preclinical AD versus normal } & \multicolumn{4}{|c|}{ Prodromal AD versus normal } & \multicolumn{4}{|c|}{ AD dementia versus normal } \\
\hline & cutoff & SE, \% & $\mathrm{SP}, \%$ & AUC & cutoff & $\mathrm{SE}, \%$ & $\mathrm{SP}, \%$ & AUC & cutoff & $\mathrm{SE}, \%$ & $\mathrm{SP}, \%$ & AUC \\
\hline $\mathrm{CSF} A \beta_{1-42}, \mathrm{pg} / \mathrm{mL}$ & $<833.0$ & 92.9 & 86.3 & 0.947 & $<752.0$ & 88.9 & 93.2 & 0.961 & $<618.4$ & 80.6 & 100.0 & 0.971 \\
\hline CSF t-Tau, pg/mL & $>253.5$ & 71.4 & 65.8 & 0.659 & $>276.2$ & 59.3 & 74.0 & 0.657 & $>343.5$ & 74.2 & 89.0 & 0.912 \\
\hline CSF p-Tau ${ }^{181}, \mathrm{pg} / \mathrm{mL}$ & $>49.9$ & 71.4 & 69.9 & 0.653 & $>49.8$ & 55.6 & 69.9 & 0.634 & $>56.2$ & 71.0 & 82.2 & 0.819 \\
\hline $\mathrm{A} \beta_{1-42} / \mathrm{A} \beta_{1-40}$ & $<0.095$ & 64.3 & 68.5 & 0.705 & $<0.095$ & 85.2 & 68.5 & 0.777 & $<0.095$ & 71.0 & 68.5 & 0.735 \\
\hline $\mathrm{t}-\mathrm{Tau} / \mathrm{A} \beta_{1-42}$ & $>0.295$ & 85.7 & 82.2 & 0.871 & $>0.295$ & 77.8 & 82.2 & 0.864 & $>0.370$ & 93.5 & 93.2 & 0.988 \\
\hline p-Tau ${ }^{181} / \mathrm{A} \beta_{1-42}$ & $>0.055$ & 78.6 & 83.6 & 0.881 & $>0.055$ & 81.5 & 83.6 & 0.894 & $>0.075$ & 90.3 & 97.3 & 0.981 \\
\hline
\end{tabular}

$\mathrm{A} \beta$, beta-amyloid protein; AD, Alzheimer's disease; AUC, area under the curve; CSF, cerebrospinal fluid; p-Tau $^{181}$, tau protein phosphorylated at threonine 181; SE, sensitivity; SP, specificity; t-Tau, total tau protein.

patients versus amyloid-negative normal subjects were $618.4 \mathrm{pg} / \mathrm{mL}$ for $A \beta_{1-42}, 343.5 \mathrm{pg} / \mathrm{mL}$ for $\mathrm{t}-\mathrm{Tau}, 56.2 \mathrm{pg} / \mathrm{mL}$ for $\mathrm{t}-\mathrm{Tau}{ }^{181}, 0.095$ for $\mathrm{A} \beta_{1-42} / \mathrm{A} \beta_{1-40}, 0.370$ for $\mathrm{t}-\mathrm{Tau} / \mathrm{A} \beta_{1-42}$, and 0.075 for $\mathrm{p}-\mathrm{Tau}{ }^{181} / \mathrm{A} \beta_{1-42}$. These cutoff values had very good sensitivity, specificity, and AUC in each group comparison (Table 4). The comparison data of preclinical AD and prodromal AD patients versus normal amyloid-negative subjects are also shown in Table 4 .

\section{Discussion}

In this study, we aimed to evaluate the usefulness of CSF biomarkers for differentiating prodromal $\mathrm{AD}$ from amyloid-negative aMCI patients in a clinically diagnosed aMCI group, and to determine new cutoff values for CSF biomarkers between the two groups. The new cutoff values for differentiating prodromal $A D$ from amyloid-negative aMCI for $A \beta_{1-42}(<749.5 \mathrm{pg} /$ $\mathrm{mL}$ ) and $\mathrm{p}-\mathrm{Tau}^{181}(>43.5 \mathrm{pg} / \mathrm{mL}$ ) showed good sensitivity and specificity; however, the difference in values of $\mathrm{t}$-Tau and $\mathrm{A} \beta_{1-40}$ was not significant to enable differentiating between the two groups. The ratios of $A \beta_{1-42} / A \beta_{1-40}(<0.095), t-T a u / A \beta_{1-42}(>0.298)$, and $p-T a{ }^{181} /$ $\mathrm{A} \beta_{1-42}(>0.059)$ also showed good sensitivity and specificity, and the combination values of $\mathrm{A} \beta_{1-42}+\mathrm{p}-\mathrm{Tau}^{181}$ and $\mathrm{p}-\mathrm{Tau}{ }^{181} / \mathrm{A} \beta_{1-42}+\mathrm{t}$-Tau were more accurate than individual CSF biomarkers (Table 3).

CSF biomarkers have become accepted and adopted to varying degrees in clinical trials or the diagnosis of AD dementia worldwide. Previous studies focusing on CSF biomarkers have grouped MCI patients depending on the follow-up results, i.e., whether subjects had converted to dementia or not, instead of depending on amyloidosis at baseline. However, CSF biomarker studies of MCI patients depending on amyloidosis have been rarely reported. To our knowledge, this is the first report that allows differentiating individual with prodromal AD from amyloid-negative aMCI ones. Our findings will be helpful for selecting prodromal AD in clinically diagnosed aMCI groups, which are very heterogeneous. The correct prediction of the MCI outcome is an important point for the prevention of dementia and for opting for appropriate clinical trials that use new disease-modifying agents. The role of CSF biomarkers seems to be crucial in a routine clinical setting for MCI patients, and our results confirm this concept. Our results are expected to become increasingly important in the future.

We also determined new cutoff values for CSF biomarkers that differentiate amyloidpositive AD dementia patients $(n=31)$ from amyloid-negative normal subjects $(n=73)$. Our individual cutoff values for t-Tau $(>343.5 \mathrm{pg} / \mathrm{mL})$ and $\mathrm{p}-\mathrm{Tau}^{181}(>56.2 \mathrm{pg} / \mathrm{mL})$ were similar 
to those reported in previous studies [34-36], but that for $\mathrm{A} \beta_{1-42}(<618.4 \mathrm{pg} / \mathrm{mL})$ was higher than that reported in previous studies $(<481-550 \mathrm{pg} / \mathrm{mL})$ [34-36]. These findings may have resulted from the fact that our normal amyloid-negative group did not include amyloidpositive normal individuals that may have been included in previous studies. Many previous studies stated that the CSF biomarkers $A \beta_{1-42}$, t-Tau, and $\mathrm{p}$-Tau could differentiate between AD dementia patients and normal healthy controls as well as between the MCI conversion and MCI stable groups, on the basis of clinical diagnosis instead of the status of amyloidosis. Because of that, our data showed some different cutoff values of CSF biomarkers compared to those of previous studies.

We also analyzed CSF biomarkers between normal amyloid-negative subjects ( $n=73$ ) and preclinical AD patients $(n=14)$. Our results suggested that new cutoff values for CSF $\mathrm{A} \beta_{1-42}(<833.0 \mathrm{pg} / \mathrm{mL})$ and $\mathrm{t}-\mathrm{Tau}(>253.5 \mathrm{pg} / \mathrm{mL})$ provided good sensitivity and specificity for differentiating preclinical AD patients from the normal amyloid-negative group. The mean concentration of CSF A $\beta_{1-42}(1,047.5 \pm 186.6 \mathrm{pg} / \mathrm{mL}, 643.0 \pm 171.1 \mathrm{pg} / \mathrm{mL})$, t-Tau $(237.8 \pm$ $97.2 \mathrm{pg} / \mathrm{mL}, 300.6 \pm 132.0 \mathrm{pg} / \mathrm{mL}$ ), and p-Tau ${ }^{181}(46.2 \pm 17.1 \mathrm{pg} / \mathrm{mL}, 55.1 \pm 20.9 \mathrm{pg} / \mathrm{mL})$ of amyloid-negative normal subjects and preclinical AD patients in our data (Table 2) is very similar to the results $\left(A \beta_{1-42}: 1,058.7 \pm 338.3\right.$ vs. $612.5 \pm 201.29 \mathrm{pg} / \mathrm{mL}$; $\mathrm{t}-\mathrm{Tau}: 255.4 \pm 104.3$ vs. $382.3 \pm 114.6 \mathrm{pg} / \mathrm{mL}^{\mathrm{p}}$-Tau ${ }^{181}: 45.1 \pm 14.6$ vs. $62.3 \pm 12.3 \mathrm{pg} / \mathrm{mL}$ ) of amyloid-negative $(n=35)$ and amyloid-positive $(n=16)$ normal subjects on the status of $\mathrm{A} \beta$ deposition reported by Dubois et al. [37]. According to these results, there may be no racial differences of CSF biomarkers in cognitively normal subjects because of amyloidosis.

In this study, CSF biomarkers reflect the progression of $\mathrm{AD}$, following each stage of the transition of normal elderly people to AD dementia (Fig. 2).

The present study showed that the APOE $\varepsilon 4$ allele frequency is highest in AD dementia patients among the three clinical diagnostic groups. It is also higher in the MCI group than in normal elderly people (Table 1 ). The APOE $\varepsilon 4$ frequency in the prodromal AD group is similar to that in the amyloid-positive AD dementia group. On the other hand, the APOE $\varepsilon 4$ frequency in amyloid-negative aMCI patients is similar to that in amyloid-negative normal subjects (Table 2). Previous studies reported that there was a significant association of APOE $\varepsilon 4$ allele and late-onset $A D$, and the APOE $\varepsilon 4$ frequency was significantly higher in sporadic AD patients than in controls [38]. APOE $\varepsilon 4$ is a very strong risk factor for AD in the cohort study and lowers age at onset of late-onset AD significantly [39]. The APOE $\varepsilon 4$ allele is associated with the molecular pathway that promotes late-onset AD [40] and is a risk factor not only for lateonset $\mathrm{AD}$, but also for early-onset $\mathrm{AD}$ [41]. Our results showed that the APOE $\varepsilon 4$ allele also bears strong association with amyloidosis in preclinical AD and prodromal AD as well as AD dementia, and that it is a strong predictor of amyloidosis in patients with aMCI and preclinical AD. Our findings are important for ongoing and future clinical trials involving participants with prodromal AD.

Overall, cognitive performance on all tests did not distinguish prodromal AD from amyloid-negative aMCI in the aMCI group (online suppl. Table 1 ). This result suggests that $A \beta$ depositions are not severe enough to affect cognitive function or are compensated by brain changes in patients with aMCI.

\section{Conclusion}

When considering the sensitivity, specificity, reliability, costs, and invasiveness of different biomarkers, we propose that the described CSF biomarkers be used as a first-line choice for the differentiation of prodromal AD in clinically diagnosed aMCI patients or for selecting very early $\mathrm{AD}$ dementia to be treated with the new disease-modifying agents. 
Accordingly, the introduction of CSF examination as a routine diagnostic procedure for selecting prodromal AD should be considered in the net decisions of diagnostic guidelines for $\mathrm{AD}$ dementia. CSF biomarkers, especially $\mathrm{A} \beta_{1-42}$ and $\mathrm{p}-\mathrm{Tau}{ }^{181}$, could be a differentiating marker for the detection of prodromal AD from clinically diagnosed aMCI patients.

\section{Acknowledgments}

This study was supported by the Brain Research Program through the National Research Foundation of Korea funded by the Ministry of Science, ICT \& Future Planning (K.H. Lee and B.C. Kim: NRF-2014M3C7A1046041; J.S. Lee: NRF-2016M3C7A1905469; B.C. Kim: NRF2016M3C7A1905472).

\section{Statement of Ethics}

Two centers in Gwangju City, Korea (Chonnam National University Hospital and Chosun University Hospital) participated in the study. The study design was approved by the Ethics Committees of Chonnam National University Hospital and Chosun University Hospital, Gwangju, Korea. All participants provided written informed consent at the time of inclusion in the cohort for use of data, samples, and images, and all study procedures were approved by the Institutional Review Boards at the Chonnam National University Hospital and Chosun University Hospital.

\section{Disclosure Statement}

The authors have no conflicts of interest to declare.

\section{Author Contributions}

B.C. Kim and K.H. Lee provided overall project supervision and directed the study. J.E. Park and K.Y. Choi contributed to the analysis and interpretation of data and drafting. S.-M. Choi, M.-K. Song, J.J. Lee, and H.-W. Kim contributed to the clinical data collection. J. Kim, H.-C. Song, and J.-M. Ha contributed to the interpretation of the amyloid PET images. E.H. Seo contributed to the analysis of neuropsychological data. W.K. Song and S.-G. Park contributed to the analysis and interpretation of laboratory data. J.E. Park and J.S. Lee led the analysis and interpretation of CSF assays. All authors edited the manuscript for content and approved its final version.

\section{References}

1 Reisberg B, Ferris SH, de Leon MJ, Crook T. The Global Deterioration Scale for assessment of primary degenerative dementia. Am J Psychiatry. 1982 Sep;139(9):1136-9.

2 Petersen RC. Mild cognitive impairment as a diagnostic entity. J Intern Med. 2004 Sep;256(3):183-94.

3 Winblad B, Palmer K, Kivipelto M, Jelic V, Fratiglioni L, Wahlund LO, et al. Mild cognitive impairment - beyond controversies, towards a consensus: report of the International Working Group on Mild Cognitive Impairment. J Intern Med. 2004 Sep;256(3):240-6. 
4 Petersen RC, Roberts RO, Knopman DS, Boeve BF, Geda YE, Ivnik RJ, et al. Mild cognitive impairment: ten years later. Arch Neurol. 2009 Dec;66(12):1447-55.

5 Tifratene K, Robert P, Metelkina A, Pradier C, Dartigues JF. Progression of mild cognitive impairment to dementia due to AD in clinical settings. Neurology. 2015 Jul;85(4):331-8.

6 Vos SJ, van Rossum IA, Verhey F, Knol DL, Soininen H, Wahlund LO, et al. Prediction of Alzheimer disease in subjects with amnestic and nonamnestic MCI. Neurology. 2013 Mar;80(12):1124-32.

7 Ravaglia G, Forti P, Maioli F, Martelli M, Servadei L, Brunetti N, et al. Conversion of mild cognitive impairment to dementia: predictive role of mild cognitive impairment subtypes and vascular risk factors. Dement Geriatr Cogn Disord. 2006;21(1):51-8.

8 Busse A, Hensel A, Gühne U, Angermeyer MC, Riedel-Heller SG. Mild cognitive impairment: long-term course of four clinical subtypes. Neurology. 2006 Dec;67(12):2176-85.

9 Ding D, Zhao Q, Guo Q, Liang X, Luo J, Yu L, et al.; Shanghai Aging Study (SAS). Progression and predictors of mild cognitive impairment in Chinese elderly: A prospective follow-up in the Shanghai Aging Study. Alzheimers Dement (Amst). 2016 Apr; 4:28-36.

10 Albert MS, DeKosky ST, Dickson D, Dubois B, Feldman HH, Fox NC, et al. The diagnosis of mild cognitive impairment due to Alzheimer's disease: recommendations from the National Institute on Aging-Alzheimer's Association workgroups on diagnostic guidelines for Alzheimer's disease. Alzheimers Dement. 2011 May; $7(3): 270-9$.

11 Dubois B, Feldman HH, Jacova C, Cummings JL, Dekosky ST, Barberger-Gateau P, et al. Revising the definition of Alzheimer's disease: a new lexicon. Lancet Neurol. 2010 Nov;9(11):1118-27.

12 Vos SJ, Verhey F, Frölich L, Kornhuber J, Wiltfang J, Maier W, et al.; Alzheimer's Disease Neuroimaging Initiative. Prevalence and prognosis of Alzheimer's disease at the mild cognitive impairment stage. Brain. 2015 May; 138(Pt 5):1327-38.

13 Dubois B, Feldman HH, Jacova C, Hampel H, Molinuevo JL, Blennow K, et al. Advancing research diagnostic criteria for Alzheimer's disease: the IWG-2 criteria. Lancet Neurol. 2014 Jun;13(6):614-29.

14 Vandermeeren M, Mercken M, Vanmechelen E, Six J, van de Voorde A, Martin JJ, et al. Detection of tau proteins in normal and Alzheimer's disease cerebrospinal fluid with a sensitive sandwich enzyme-linked immunosorbent assay. J Neurochem. 1993 Nov;61(5):1828-34.

15 Tapiola T, Alafuzoff I, Herukka SK, Parkkinen L, Hartikainen P, Soininen H, et al. Cerebrospinal fluid \{beta\}amyloid 42 and tau proteins as biomarkers of Alzheimer-type pathologic changes in the brain. Arch Neurol. 2009 Mar; 66(3):382-9.

16 Haense C, Buerger K, Kalbe E, Drzezga A, Teipel SJ, Markiewicz P, et al. CSF total and phosphorylated tau protein, regional glucose metabolism and dementia severity in Alzheimer's disease. Eur J Neurol. 2008 Nov; 15(11):1155-62.

17 Hampel H, Goernitz A, Buerger K. Advances in the development of biomarkers for Alzheimer's disease: from CSF total tau and Abeta(1-42) proteins to phosphorylated tau protein. Brain Res Bull. 2003 Aug;61(3):243-53.

18 Sarker I, Khan RK, Haque A, Hannan MA, Islam R, Rizvi AN, Helal AH, Biswas SK. Association of cerebrospinal fluid tau protein in patients with Alzheimer's and Non Alzheimer's dementias in a tertiary level hospital in Bangladesh. Int J Clin Exp Neurol. 2017;5(1):11-17.

19 Waldemar G, Dubois B, Emre M, Georges J, McKeith IG, Rossor M, et al.; EFNS. Recommendations for the diagnosis and management of Alzheimer's disease and other disorders associated with dementia: EFNS guideline. Eur J Neurol. 2007 Jan;14(1):e1-26.

20 Hughes CP, Berg L, Danziger WL, Coben LA, Martin RL. A new clinical scale for the staging of dementia. Br J Psychiatry. 1982 Jun;140:566-72.

21 Morris JC. The Clinical Dementia Rating (CDR): current version and scoring rules. Neurology. 1993 Nov; 43(11):2412-4.

22 Lawton MP, Brody EM. Assessment of older people: self-maintaining and instrumental activities of daily living. Gerontologist. 1969;9(3):179-86.

23 Ku HM, Kim JH, Kwon EJ, Kim SH, Lee HS, Ko HJ, et al. A study on the reliability and validity of Seoul-Instrumental Activities of Daily Living (S-IADL). J Korean Neuropsychiatr Assoc. 2004;43:189-99.

24 Folstein MF, Folstein SE, McHugh PR. "Mini-mental state." A practical method for grading the cognitive state of patients for the clinician. J Psychiatr Res. 1975 Nov;12(3):189-98.

25 Kang Y, Na DL, Hahn S: A validity study on the Korean Mini-Mental State Examination (K-MMSE) in dementia patients. J Korean Neurol Assoc. 1997;15(2):300-8.

26 Kang Y, Na DL. Seoul Neuropsychological Screening Battery (SNSB). Seoul: Human Brain Research \& Consulting Co; 2003.

27 Noh Y, Lee Y, Seo SW, Jeong JH, Choi SH, Back JH, et al. A new classification system for ischemia using a combination of deep and periventricular white matter hyperintensities. J Stroke Cerebrovasc Dis. 2014 Apr;23(4): 636-42.

28 McKhann GM, Knopman DS, Chertkow H, Hyman BT, Jack CR Jr, Kawas CH, et al. The diagnosis of dementia due to Alzheimer's disease: recommendations from the National Institute on Aging-Alzheimer's Association workgroups on diagnostic guidelines for Alzheimer's disease. Alzheimers Dement. 2011 May;7(3):263-9.

29 Becker GA, Ichise M, Barthel H, Luthardt J, Patt M, Seese A, et al. PET quantification of 18F-florbetaben binding to $\beta$-amyloid deposits in human brains. J Nucl Med. 2013 May;54(5):723-31. 
Park et al.: CSF Biomarkers for the Diagnosis of Prodromal AD

30 Barthel H, Luthardt J, Becker G, Patt M, Hammerstein E, Hartwig K, et al. Individualized quantification of brain $\beta$-amyloid burden: results of a proof of mechanism phase 0 florbetaben PET trial in patients with Alzheimer's disease and healthy controls. Eur J Nucl Med Mol Imaging. 2011 Sep;38(9):1702-14.

31 Patt M, Schildan A, Barthel H, Becker G, Schultze-Mosgau M, Rohde B, et al. Metabolite analysis of [18F] Florbetaben (BAY 94-9172) in human subjects: a substudy within a proof of mechanism clinical trial. J Radioanal Nucl Chem. 2010;284(3):557-62.

32 Sabri O, Seibyl J, Rowe C, Barthel H. Beta-amyloid imaging with florbetaben. Clin Transl Imaging. 2015;3(1): 13-26.

33 DeLong ER, DeLong DM, Clarke-Pearson DL. Comparing the areas under two or more correlated receiver operating characteristic curves: a nonparametric approach. Biometrics. 1988 Sep;44(3):837-45.

34 Park SA, Chae WS, Kim HJ, Shin HS, Kim S, Im JY, et al. Cerebrospinal Fluid Biomarkers for the Diagnosis of Alzheimer Disease in South Korea. Alzheimer Dis Assoc Disord. 2017 Jan-Mar;31(1):13-8.

35 Mulder C, Verwey NA, van der Flier WM, Bouwman FH, Kok A, van Elk EJ, et al. Amyloid-beta(1-42), total tau, and phosphorylated tau as cerebrospinal fluid biomarkers for the diagnosis of Alzheimer disease. Clin Chem. 2010 Feb;56(2):248-53.

36 Mattsson N, Zetterberg H, Hansson O, Andreasen N, Parnetti L, Jonsson M, et al. CSF biomarkers and incipient Alzheimer disease in patients with mild cognitive impairment. JAMA. 2009 Jul;302(4):385-93.

37 Dubois B, Epelbaum S, Nyasse F, Bakardjian H, Gagliardi G, Uspenskaya O, et al.; INSIGHT-preAD study group. Cognitive and neuroimaging features and brain $\beta$-amyloidosis in individuals at risk of Alzheimer's disease (INSIGHT-preAD): a longitudinal observational study. Lancet Neurol. 2018 Apr;17(4):335-46.

38 Strittmatter WJ, Saunders AM, Schmechel D, Pericak-Vance M, Enghild J, Salvesen GS, et al. Apolipoprotein E: high-avidity binding to beta-amyloid and increased frequency of type 4 allele in late-onset familial Alzheimer disease. Proc Natl Acad Sci USA. 1993 Mar;90(5):1977-81.

39 Sando SB, Melquist S, Cannon A, Hutton ML, Sletvold O, Saltvedt I, et al. APOE epsilon 4 lowers age at onset and is a high risk factor for Alzheimer's disease; a case control study from central Norway. BMC Neurol. 2008 Apr; $8(1): 9$.

40 Rhinn H, Fujita R, Qiang L, Cheng R, Lee JH, Abeliovich A. Integrative genomics identifies APOE $\varepsilon 4$ effectors in Alzheimer's disease. Nature. 2013 Aug;500(7460):45-50.

41 Genin E, Hannequin D, Wallon D, Sleegers K, Hiltunen M, Combarros O, et al. APOE and Alzheimer disease: a major gene with semi-dominant inheritance. Mol Psychiatry. 2011 Sep;16(9):903-7. 